\title{
Vacancy Graphite as the Cathode Materials of Al-Ion Batteries using First Principle Calculation
}

\author{
Faizatul Azwa Zamri, Muhamad Husaini Abu Bakar
}

\begin{abstract}
The defect graphite will change the electrochemical properties of the aluminium-ion batteries. However, the theoretical research on the defect-free graphite as cathode material for aluminium-ion batteries remain uncertain. Therefore, the objective of this paper is to develop the theoretical prediction of defect graphite to be used in the aluminium-ion batteries analysis. The structural properties of graphite and vacancy point defect of graphite were calculated using the first principle calculation. The generalized gradient approximation and van der Waals correction ( $v d W-D 3)$ implemented to the calculation. The validation of the methodology on the defect-free graphite is evaluated with the experiment and other theoretical prediction. After that, the lattice constants of the defect graphite were evaluated and calculate the formation energy. The results show that the lattice constant of defect-free graphite was closer to the experimental values compared to other theoretical prediction. However, the atomic distances near to the vacancy point observed slightly lower than other theoretical prediction using different exchange correlation approximation. The formation energy calculated for monovacancy and divacancy was $7.92 \mathrm{eV}$ and 7.34 $e V$, respectively. As a conclusion, the structural properties obtained in this calculation could be references in the development of the defect cathode analysis in the aluminium ion batteries.
\end{abstract}

Index Terms: Structural properties, graphite, defect graphite, first principle calculation

\section{INTRODUCTION}

Graphite consist of more than one layers of carbon atoms. The carbon atoms in a layer are covalently bond and the layers stacked by the Van der Waals force. Since the bonding between layer is weak, it can accommodate ions and molecules to form graphite intercalation compound (GIC) which display exclusive properties [7]. Thus, graphite material was an ideal material, especially battery cathode.

The Defect-free of cathode results in the excellent electrochemical performance mainly due to three advantages:

Revised Manuscript Received on October 30, 2019.

* Correspondence Author

Faizatul Azwa Zamri*, System Engineering and Energy Laboratory (SEELAB), Universiti Kuala Lumpur - Malaysian Spanish Institute, Kulim Hi-Tech Park, Malaysia.

Muhamad Husaini Abu Bakar, Head of Research and Innovation, System Engineering and Energy Laboratory (SEELAB), Universiti Kuala Lumpur - Malaysian Spanish Institute, Kulim Hi-Tech Park, Malaysia.

(C) The Authors. Published by Blue Eyes Intelligence Engineering and Sciences Publication (BEIESP). This is an open access article under the CC BY-NC-ND license (http://creativecommons.org/licenses/by-nc-nd/4.0/)
(1) the fast intercalation of the large size anions occurred and provide more active sites for energy storage when eliminating inactive defect; (2) empowered electrical conductivity recovering defective graphene into electrical highway; and (3) high reproducibility of material quality and cell performance favoring large scale manufacture [8]. However, it is impossible to produce defect-free graphite in large scale manufacturing, but this can reduce by specific parameter optimization. Therefore, it is vital to understand the properties of defect graphite, which potentially influenced the battery mechanism.Numbers of research has performed on the structural properties of graphite and its defect, either experimental or theoretical. The simplest point defect graphite reported due to radiation damages such as vacancy and interstitials defects [1]-[3]. The vacancy defect of graphite including monovacancy and divacancy. These structural properties had been investigated by previous research using density functional theory (DFT) within GGA [5], and LDA [3] framework. The basis set such as DNP [5] and Norm-conserving pseudopotential (NCPP) [3] are used to describe the electron wavefunction. Recently, the projector augmented wave (PAW) method used to treat the interaction between core and valance electrons in the first principle calculation for theoretical prediction of graphite properties. Besides, the Van der Waals correction, including vdW-D2 [9], vdW-D3 [10] was beneficial in the correction of potential energy and interatomic forces. The structural properties of graphite and monovacancies graphite had been elucidated with vdW-D and vdW-D2 [17] implementation but vdW-D3 implementation in the defect graphite is still unclear.Hence, the details of structural analysis of graphite structure ab-initio DFT calculation with the implementation of van der Waals correction and the comparison in term of lattice constant were presenting in this paper. After that, the structural properties of the monovacancy and the divacancy defect using the proposed calculation method were elucidated in this paper. Lastly, the formation energy of the vacancy graphite was calculated as references for future aluminium-ion batteries cathodes analysis.

\section{COMPUTATIONAL DETAILS AND METHOD}

\section{A) Computational method}

The 64 carbon atoms of the graphite were developed using Avogadro atomic modelling software to provide structural information for the model. 
The structure information, including lattice, and cartesian coordinate required in the construction of Abinit input file. The properties such as k-points and symmetries, the basis set and self-consistency procedure also described in the input file prior begin the simulation. The successful calculation produced output file, and then this was visualized using Avogadro software. Besides, the output parameter was extracted using Abipy python command.

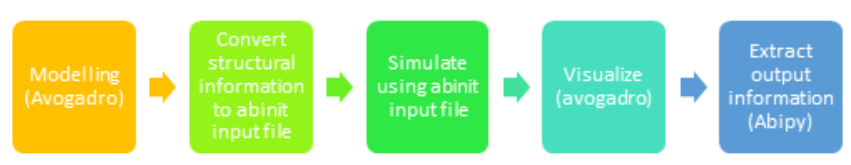

Figure 1. Computation process flow

\section{B) Structural optimization}

In this paper, the projector augmented wave (PAW) method based on the density functional theory (DFT) implemented to perform the first principle calculation using open-source Abinit software [11]-[13]. Besides, the generalized gradient approximation of Perdew-Burke-Ernzerhof (GGA-PBE) [15] is used to represent the exchange-correlation. Moreover, the DFT-D3 approach implemented for the van der Waals correction.The geometry optimization obtained using the Broyden-Fletcher-Goldfarb-Shanno minimization (BFGS) method. Total $381 \mathrm{eV}$ of the energy cut-off for the plane waves with $6 \times 6 \times 1$ of the Morhost-pack k-point grid applied to the system. The atomic and lattice position is considered by fully relaxed until the forces on all atoms are smaller than $0.012 \mathrm{eV} \AA-1$. The convergence criteria for total energy calculation customized to $1 \times 10-3$ eV.The calculation performed on the defect-free graphite consists of two layers of the graphene sheet. This $\mathrm{AB}$ stacking of hexagonal graphite consists of 64 carbon atoms. The optimized lattice constant of the structure validated with the experimental and other theoretical prediction. After that, the calculation performed on the monovacancy and divacancy defect of graphite. The formation energy also calculated for the defected structure.

\section{The formation energy}

The formation energy for vacancy graphite is determined using equation (1) based on the unrestricted spin calculation of DFT calculation. The ED, and EG are the total energies of defected and defect-free graphite, respectively. Meanwhile, N gives the number of atoms added $(+\mathrm{N})$ or removed $(-\mathrm{N})$ and $\mu$ is the chemical potential of carbon.

$\mathrm{Ef}=\mathrm{E}_{\mathrm{D}}-\mathrm{E}_{\mathrm{G}}-\mathrm{N} \mu$

\section{RESULTS AND DISCUSSIONS}

\section{A) Lattice constants}

The equilibrium structure is shown in figure 2. The C-C bond length and the interlayer distance between graphene layers calculated in this paper was $1.42 \AA$ and $3.333 \AA$, respectively which this value is in close agreement with the experimentally reported values (1.42 $\AA$, $3.336 \AA$ ) [1]. This values also close to other theoretical prediction with the value $3.4 \AA$ [5], [14].
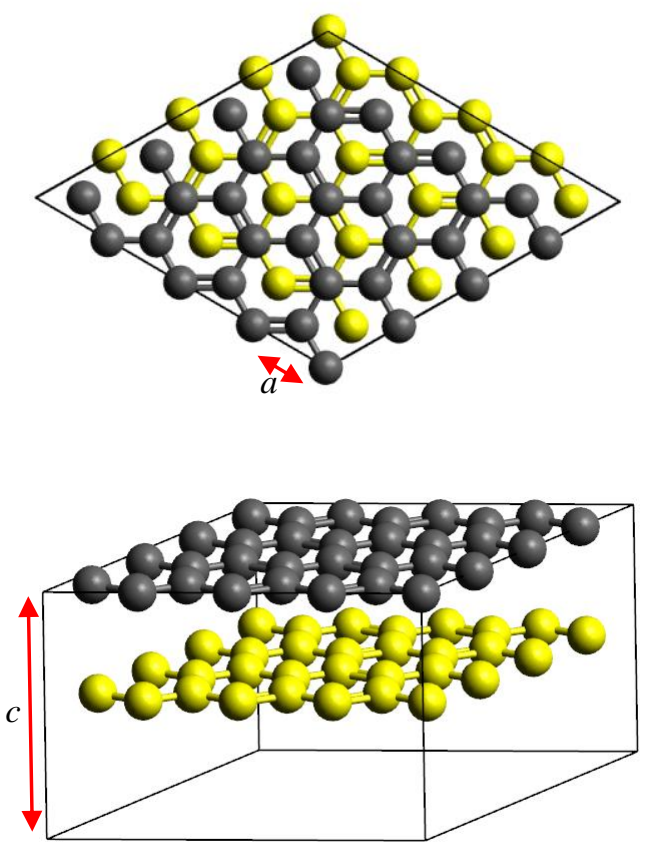

Figure 2. The equilibrium structure of 64 carbon atom graphite.

The hexagonal lattice size for $a$ and c were $2.463 \AA$ and 6.66 $\AA$, respectively as summarized in table 1 . The percentage errors calculated were $0.08 \%$ and $0.72 \%$, which compare to the experiment values was $2.461 \AA$, $6.708 \AA$, respectively. This is in agreement with the other theoretical calculation using GGA-PBE functional and vdW-D3[16], where the lattice size $a$ and $c$ was $2.467 \AA$ and $6.965 \AA$, as well as the percentage error was $0.16 \%$ and $3.83 \%$, respectively. Nevertheless, the percentage error of lattice geometry without van der Waals correction was quite high where the a and c calculated was $2.47 \AA$ and $8.68 \AA$ with $0.37 \%$ and $29.4 \%$, respectively.

Table 1: Lattice constants for hexagonal graphite structure

\section{Functional $\backslash$ Lattice}

GGA-PBE, vdW-D3 a

$2.463(0.08 \%)$ c

$6.66(0.72 \%)$

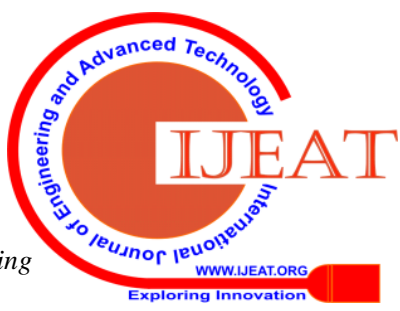




\section{B) Vacancy defects}

Firstly, the total energy calculation was performed for configuration of monovacancy at 1, 2 and 3, as shown in figure 2(a), (b) and (c), respectively. This is observed that removing atom 1 (figure 2(a)) has the lowest energy and considered in the further calculation. The equilibrium configuration of the monovacancy and divacancy hexagonal graphite as shown in figure 2(d) and 2(e) respectively. This structure observed was similar to others theoretical study [5].
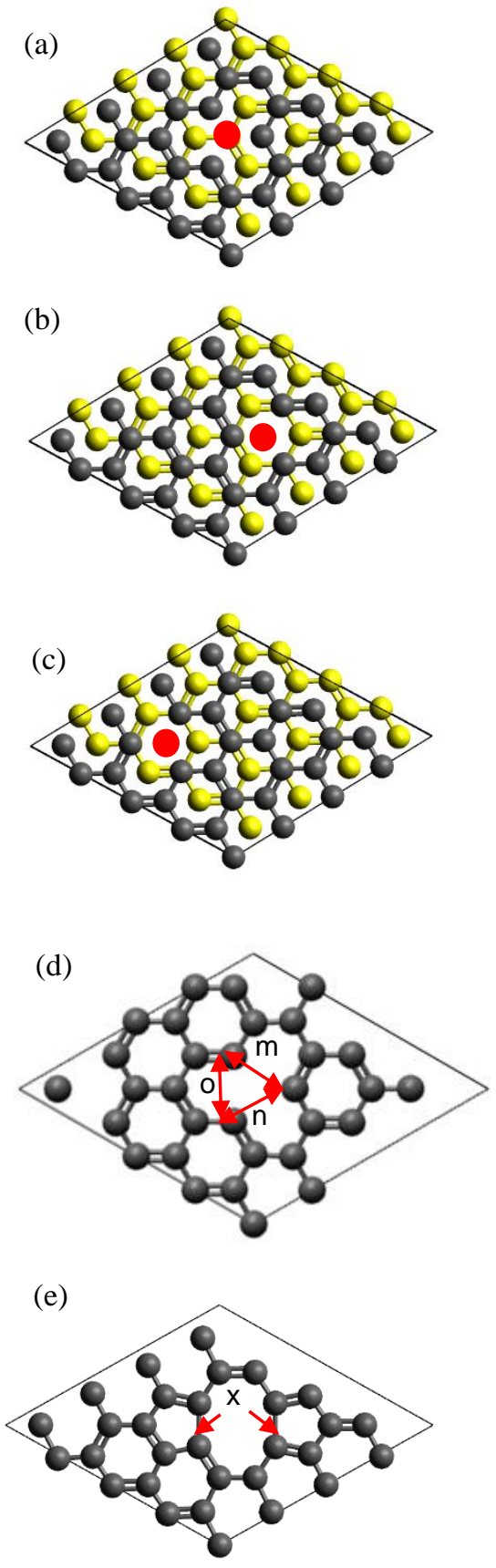

Figure 2 (a-c) configurations of point defect, and the equilibrium (d) monovacancy (e) divacancy of the hexagonal graphite.

The comparison of the atomic distances labelled as $\mathrm{m}, \mathrm{n}$, and o for monovacancy defect as well as $\mathrm{x}$ for divacancy defect of the hexagonal graphite using GGA-PBE and GGA-PW91 approximation, respectively was summarized in the table 2 . Generally, the atomic distances for this calculation was lowest than others theoretical prediction.

The monovacancy in a layer graphite experiences Jahn-Teller distortion [5], where adjacent atom of monovacancy moved closer and then forming pentagon like structure. The distance between atom 1 and 2 shortened from $2.46 \AA$ to $2.37 \AA$. This result is in agreement with other theoretical prediction [5] but the there is single bond connecting between atom 1 and 2. In contrast, there was no bonding between atom 1 and 2 in this calculation. Nevertheless, the distance from atom 1 to 3 and 2 to 3 was expanded from $2.46 \AA$ to $2.53 \AA$ which was lower than other theoretical prediction, $2.60 \AA$ [1] and $2.63 \AA$ [5]. The divacancy is indicated by removing adjacent carbon atoms from the graphite lattice. The carbon atom near the vacancy forming pentagon-hexagonal-pentagon shapes, which is referred to the 5-8-5 topology defect [5]. The atomic distances labelled $\mathrm{x}$ was $1.74 \AA$, which is increased from the original bond length (1.42 $\AA$ ). This finding is slightly lower than other theoretical prediction, which was $1.82 \AA$.

Table 2: The atomic distances for point defects of hexagonal graphite.

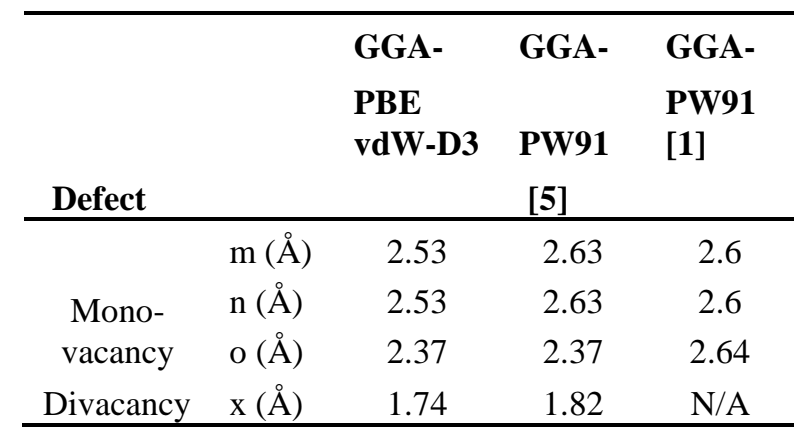

\section{C) Formation energy}

The formation energies indicate that energy required for defect to be formed. The formation energy calculated for monovacancy using GGA-PBE approximation and vdw-DW was $7.92 \mathrm{eV}$. This value is slightly higher than other GGA approximation, $7.84 \mathrm{eV}$, and $7.38 \mathrm{eV}$ for Perdew and Wang, 91 [5] and Perdew and Wang, 96 [6], respectively.

Published By: 
However, this calculation was lower than LDA with the parameterisation of Perdew and Wang [6], $7.99 \mathrm{eV}$ as tabulated in table 2.The formation energy of divacancy was lower than monovacancy defect with the value $7.34 \mathrm{eV}$. This is suggesting that divacancy was more favourability compared to the monovacancy defect. This is similar finding with others work [5].

Table 2. the formation energy of the monovacancy and divacancy defect of hexagonal graphite.

\begin{tabular}{lll}
\hline Structure & $\begin{array}{l}\text { Monovacanc } \\
\mathbf{y}(\mathbf{e V})\end{array}$ & $\begin{array}{l}\text { Divacanc } \\
\mathbf{y}(\mathbf{e V})\end{array}$ \\
\hline $\begin{array}{l}\text { GGA-PBE } \\
\text { vdW-D3 }\end{array}$ & 7.92 & 7.34 \\
GGA-PW91 & & \\
[5] & 7.84 & 7.53 \\
GGA-PBE96 & & \\
[6] & 7.38 & N/A \\
LDA-PW92 & & \\
[6] & 7.99 & N/A \\
\hline
\end{tabular}

\section{CONCLUSION}

In summary, the first principle calculation of the interacting defect-free, monovacancy and divacancy of hexagonal graphite was evaluated with GGA and vdW-D3 of the exchange correlation approximation and van der Waals correction, respectively. The optimized lattice of hexagonal graphite was $2.463 \AA(a)$ and $6.66 \AA(c)$ with the values has $0.08 \%$ and $0.72 \%$, respectively which close to the experimental results. The C-C bond length calculated $1.42 \AA$ and the interlayer distance was $3.33 \AA$. This value was in agreement with experiment and theoretical results. The atomic distances of monovacancy and divacancy calculated in this paper was slightly lower than other theoretical prediction. The formation energy was $7.92 \mathrm{eV}$ and $7.34 \mathrm{eV}$ for monovacancy and divacancy of defect graphite, respectively. Lastly, further studies about this defect required in the application of cathode materials in the aluminium-ion batteries.

\section{ACKNOWLEDGEMENT}

The work described in this paper is supported by the FRGS grant no. FRGS/1/2018/TK07/UNIKL/02/3/RA-app/2019 and System Engineering and Energy (SEELAB), UniKL, Malaysian-Spanish Institute, Kulim kedah, Malaysia.

\section{REFERENCES}

1. L. Li, S.Reich, \& Robertson, J. (2005, November). Defect energies of graphite: Density-functional calculations. Physical Review B Condensed Matter and Materials Physics. Available: https://doi.org/10.1103/PhysRevB.72.184109

2. R.H.Telling, \& M.I.Heggie, (2007,September). Radiation defects in graphite. Philosophical Magazine. 87(31). pp. 4797-4846 https://doi.org/10.1080/14786430701210023

3. A.A.Elbarbary, R.H.Telling, C.P.Ewels, C.P.Ewels, M.I.Heggie, \& P.R.Briddon (2003, May). Structure and energetics of the vacancy in graphite. Physical Review B - Condensed Matter and Materials $\begin{array}{llll}\text { Physics. } & 68 & \text { (14). } & \text { Available: }\end{array}$ https://doi.org/10.1103/PhysRevB.68.144107

4. M. T.Yin, \& M. L.Cohen, (1984, Jun). Structural theory of graphite and graphitic silicon. Physical Review B.29(12). pp.6996-6998. Available: https://doi.org/10.1103/PhysRevB.29.6996

5. H.Zhang, M. Zhao, X.Yang, H.Xia, X.Liu, \& Y.Xia, (2010,October). Diffusion and coalescence of vacancies and interstitials in graphite: A first-principles study. Diamond and Related Materials.19(10).pp.1240-1244.Available: https://doi.org/10.1016/j.diamond.2010.06.010 Condensed https://doi.org/10.1016/j.ensm.2018.04.023 Available: https://doi.org/10.1002/jcc.20495 https://doi.org/10.1063/1.3382344 Communications. https://doi.org/10.1016/j.cpc.2016.04.003 Communications.

https://doi.org/10.1016/j.cpc.2009.07.007 https://doi.org/10.1524/zkri.220.5.558.65066 Engineering. 77(18).pp.3865-3868.Available: https://doi.org/10.1103/PhysRevLett.77.3865 https://doi.org/10.1103/PhysRevB.98.174103 https://doi.org/10.1016/j.apsusc.2016.04.105 https://doi.org/10.1006/jssc.1999.8448 https://doi.org/10.1103/PhysRev.100.544

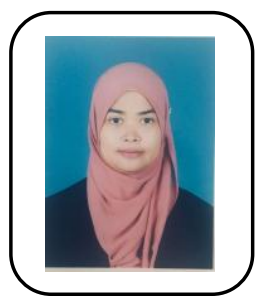
(2013,March). The contribution made by lattice vacancies to the Wigner effect in radiation-damaged graphite. Journal of Physics Matter.25(13).Available: https://doi.org/10.1088/0953-8984/25/13/135403

7. D. D. L.Chung, (2016, December). Graphite Intercalation Compounds Reference Module in Materials Science and Materials Engineering. Available: https://doi.org/10.1016/B978-0-12-803581-8.02311-0

8. M.Zhang, X.Song, X.Ou, \& Y.Tang, (2019,January). Rechargeable batteries based on anion intercalation graphite cathodes. Energy Storage Materials. 16.pp. 65-84.Available:

9. S.Grimme, (2006,November) Semiempirical GGA-type density functional constructed with a long- range dispersion correction. Journal of Computational Chemistry. 27(15). pp.1787-1799.

10. S.Grimme, J.Antony, S.Ehrlich, \& H.A.Krieg, (2010,March). Consistent and accurate ab initio parametrization of density functional dispersion correction (DFT-D) for the 94 elements H-Pu, The Journal of Chemical Physics, 132(15), pp.154104. Available:

11. X.Gonze, F.Jollet, F.Abreu Araujo, D.Adams, B.Amadon, T.Applencourt, ... J.W.Zwanziger, (2016,August). Recent developments in the ABINIT software package. Computer Physics

12. X.Gonze, B.Amadon, P.M.Anglade, J.M.Beuken, F.Bottin, P.Boulanger, J.W.Zwanziger, (2009, July). ABINIT: First-principles approach to material and nanosystem properties. Computer Physics

13. X.Gonze, G.M.Rignanese, M.Verstraete, J.M.Beuken, Y.Pouillon, R. Caracas, ... D.C.Allan, (2005, August). A brief introduction to the ABINIT software package. Zeitschrift Fur Kristallographie. Available:

14. P.Lambin, \& J.Fink, (2016,Jun). Electronic States of Carbon Materials. Reference Module in Materials Science and Materials

Available:

15. J.P.Perdew, K.Burke, \& M.Ernzerhof, (1996,October). Generalized gradient approximation made simple. Physical Review Letters.

16. I.Mosyagin, D.Gambino, D.G.Sangiovanni, I.A.Abrikosov, \& N.M.Caffrey, (2018,November). Effect of dispersion corrections on ab initio predictions of graphite and diamond properties under pressure. Physical Review $\quad$ B. $\quad$ Available:

17. J.Liu, C.Wang, T.Liang, \& W.Lai, (2016,April). Interaction of boron with graphite: A van der Waals density functional study. Applied Surface Science,379,pp.402-410.Available:

18. J.Fayos, (1999,December). Possible 3D Carbon Structures as Progressive Intermediates in Graphite to Diamond Phase Transition. Journal of Solid State Chemistry.148(2).pp.278-285.Available:

19. Y.Baskin, \& L.Meyer, (1955,October). Lattice constants of graphite at low temperatures. Physical Review. Available:

\section{AUTHORS PROFILE}

First Author Faizatul Azwa Binti Zamri is a Ph.D. candidate for System engineering and Energy Laboratory located at Universiti Kuala Lumpur Malaysian Spanish Institute, Malaysia Background Bachelor (Hons) in the Mechanical Engineering at Universiti Sains Malaysia (2010) She worked in the semiconductor company at Perak from 2010 to 2013 as process engineer. Awarded 
Master of Sciences (Material Science) from Universiti Putra Malaysia (2017). She worked as part time tutor at Universiti Putra Malaysia from 2013 to 2017. Her research interest in the quantum modelling and Energy (Aluminium ion batteries).

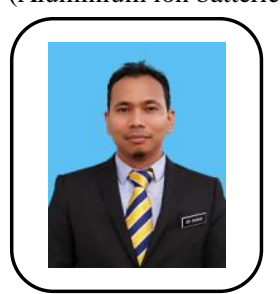

Second Author Muhamad Husaini Abu Bakar is Director for System engineering and Energy Laboratory and Head of Research and Innovation Section at the Universiti Kuala Lumpur Malaysian Spanish Institute,Malaysia. Having obtained a Bachelor Degree in Manufacturing Engineering with Management at the Universiti Sains Malaysia (2007), Malaysia, he spent the time from 2007-2012 at the Underwater Robotic Research Group, Universiti Sains Malaysia as a research engineer and awarded Master of Science in advance manufacturing from Universiti Sains Malaysia in 2011. Since 2012 he work as lecturer at Universiti Kuala Lumpur - Malaysian Spanish Institute and obtain his Doctor of Philosophy in Advanced Manufacturing from Universiti Sains Malaysia in 2017. His research interests are related to smart manufacturing, energy, and atomistic modelling. He has published over 50 scientific publications in Deep Learning,computational atomistic and metal-air battery. His research activities were recognized by the innovation award of PECIPTA, IIDEX, ICOMPEX, I-ENVEX and MARA due to his innovative invention in smart material and smart monitoring system. 